\title{
NONLOCAL EIGENVALUE PROBLEMS WITH INDEFINITE WEIGHT
}

\author{
SAID TAARABTI
}

\begin{abstract}
In the present paper, we consider a class of eigenvalue problems driven by a nonlocal integro-differential operator $\mathcal{L}_{K}^{p(x)}$ with Dirichlet boundary conditions. Under certain assumptions on p and $\mathrm{q}$, we establish that any $\lambda>0$ suficiently small is an eigenvalue of the nonhomogeneous nonlocal problem $\left(\mathcal{P}_{\lambda}\right)$.

Розглядається клас спектральних задач, пов'язаних із нелокальним інтегродиференціальним оператором $\mathcal{L}_{K}^{p(x)}$ із крайовою умовою Дирихле. За певних припущень щодо $p$ i $q$ доведено, що кожне достаньо мале $\lambda>0$ є власним значенням неоднорідної нелокальної задачі $\left(\mathcal{P}_{\lambda}\right)$.
\end{abstract}

\section{INTRODUCTION}

Let $\Omega \subset \mathbb{R}^{N}, N \geq 2$, be a bounded regular open domain and consider the following problem involving the Fractional $p(x, y)$-Laplacian with Dirichlet boundary condition:

$$
\left(\mathcal{P}_{\lambda}\right)\left\{\begin{array}{cccccc}
\mathcal{L}_{K}^{p(x)} u+|u|^{\bar{p}(x)-2} & u & = & \lambda V(x)|u|^{q(x)-2} u & \text { in } & \Omega \\
u & = & 0 & & \text { in } & \mathbb{R}^{N} \backslash \Omega,
\end{array}\right.
$$

where $\lambda>0$ is a real number, $K$ is a suitable kernel, $\bar{p}=p(x, x), V: \Omega \longrightarrow \mathbb{R}$ is an indefinite weight function, $p: \mathbb{R}^{N} \times \mathbb{R}^{N} \longrightarrow(1,+\infty)$ is a continuous function satisfying the following assumptions:

$$
1<p^{-}=\min _{(x, y) \in \bar{\Omega} \times \bar{\Omega}} p(x, y) \leq p(x, y) \leq p^{+}=\max _{(x, y) \in \bar{\Omega} \times \bar{\Omega}} p(x, y)<+\infty
$$

$p \quad$ is symmetric, that is, $\quad p(x, y)=p(y, x) \quad \forall(x, y) \in \bar{\Omega} \times \bar{\Omega}$

and $q: \bar{\Omega} \longrightarrow(1,+\infty)$ is a bounded continuous function such that

$$
1<q^{-}=\min _{x \in \bar{\Omega}} q(x) \leq q^{+}=\max _{x \in \bar{\Omega}} q(x)<p^{-} \quad \forall x \in \bar{\Omega} .
$$

Recently, a great deal of attention has been focused on studying of problems involving integro-differential operators of nonlocal fractional type. In [25], Wenjing Chen and Shengbing Deng studied the following fractional elliptic problem

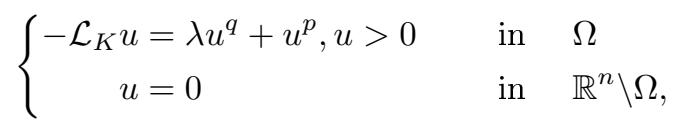

where $\mathcal{L}_{K}$ is an integro-differential operators of nonlocal fractional type defined as follows:

$$
\mathcal{L}_{K}(u)=\int_{\mathbb{R}^{N}}(u(x+y)+u(x-y)-2 u(x)) K(y) d y, \quad x \in \mathbb{R}^{n},
$$

they showed the multiplicity of solutions to equations driven by a nonlocal integrodifferential operator $\mathcal{L}_{K}$ with homogeneous Dirichlet boundary conditions.

2020 Mathematics Subject Classification. 35J20, 35S05, 35B60.

Keywords. Fractional $p(x, y)$-Laplacian problems, eigenvalue problem, Ekeland's variational principle, indefinite weight, fractional Sobolev space. 
R. Servadei and E. Valdinoci studied the following problem

$$
\begin{cases}(-\Delta)^{s} u-\lambda u=|u|^{2^{*}-2} u & \text { in } \Omega \\ u=0 & \text { in } \mathbb{R}^{n} \backslash \Omega\end{cases}
$$

where $(-\Delta)^{s}$ is the fractional Laplace operator, $s \in(0,1), \Omega$ is an open bounded set of $\mathbb{R}^{n}, n>2 s$, with Lipschitz boundary, $\lambda>0$ is a real parameter and $2^{*}=2 n /(n-2 s)$ is a fractional critical Sobolev exponent. In [29], they proved that there exists $\lambda_{s}>0$ such that for any $\lambda>\lambda_{s}$ different from the eigenvalues of $(-\Delta)^{s}$ problem (1.7) admits a weak solution $u \in H^{s}\left(\mathbb{R}^{n}\right)$, which is not identically zero, and such that $u=0$ a.e. in $\mathbb{R}^{n} \backslash \Omega$.

After that, the same authors, in [30], studied the problem in a general framework; indeed they considered the following equation

$$
\begin{cases}\mathcal{L}_{K} u+\lambda u+|u|^{2^{*}-2} u+f(x, u)=0 & \text { in } \Omega \\ u=0 & \text { in } \mathbb{R}^{n} \backslash \Omega\end{cases}
$$

where $\mathcal{L}_{K}$ is a general non-local integrodifferential operator of order $s$ and $f$ is a lower order perturbation of the critical power $|u|^{2^{*}-2} u$. In this setting they proved an existence result through variational techniques.

In the [15], the authors investigated the following Brézis-Nirenberg type problem:

$$
\left\{\begin{aligned}
\mathcal{L}_{K} u & =\mu|u|^{2^{\star}-2} u+\lambda g(u) & & \text { in } \Omega \\
u & =0 & & \text { in } \mathbb{R}^{n} \backslash \Omega,
\end{aligned}\right.
$$

they proved the existence of one weak solution of (1.8) through direct minimization of the energy in a small ball of a certain fractional Sobolev space.

Also, in [23], Nguyen Thanh Chung considered the following problem

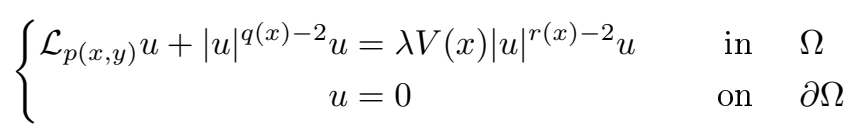

where $\mathcal{L}_{p(x, y)}$ is the fractional $p(x, y)$-Laplace operator given by

$$
\mathcal{L}_{p(x, y)} u=\left(-\Delta_{p(x)}\right)^{s}(u)=p . v \cdot \int_{\Omega} \frac{|u(x)-u(y)|^{p(x, y)-2}(u(x)-u(y))}{|x-y|^{N+s p(x, y)}} d y, \quad s \in(0,1),
$$

where $p . v$. is a commonly used abbreviation in the principal value sense. He established some results on the existence of a continuous family of eigenvalues using variational techniques and Ekeland's variational principle.

Note that the operator $\left(-\Delta_{p(x)}\right)^{s}$ is the fractional version of well known $p(x)$-Laplacian operator $\Delta_{p(x)} u(x)=\operatorname{div}\left(|\nabla u(x)|^{p(x)-2} u(x)\right)$. On the other hand, we remark that in the constant exponent case it is known as the fractional $p$-Laplacian operator $\left(-\Delta_{p}\right)^{s}$. This nonlocal nonlinear operator is consistent, up to some normalization constant depending upon $N$ and $s$, with the linear fractional Laplacian $(-\Delta)^{s}$ in the case $p=2$. The interest for this last operator and more generally pseudo-differential operators has constantly increased over the last few years, although such operators have been a classical topic of functional analysis since long ago. Nonlocal operators such as $(-\Delta)^{s}$ and its generalisation $\mathcal{L}_{K}$ like in probleme (1.8) (for more details see [26, 27, 28]) naturally arise in continuum mechanics, phase transition phenomena, population dynamics and game theory, as they are the typical outcome of stochastical stabilization of Lévy processes, see e.g. $[6,21,22]$.

The interest in studying non-local integro-differential was stimulated by their applications. Indeed, they have impressive applications in different fields, as the thin obstacle 
problem, optimization, finance, stratified materials, anomalous diffusion, crystal dislocation, deblurring and denoising of images, and so on. For further details we refer to $[7,8,9,10,20,24]$ and the references therein.

Now, we introduce the nonlocal integro-differential operator of elliptic type $\mathcal{L}_{K}^{p(x)}$ which generalizes $\left(-\Delta_{p}\right)^{s}$, for any fixed $s \in(0,1)$, as follows:

$$
\begin{aligned}
\mathcal{L}_{K}^{p(x)} u & =\mathrm{p} \cdot \mathrm{v} \cdot \int_{\mathbb{R}^{N}}|u(x)-u(y)|^{p(x, y)-2}(u(x)-u(y)) K(x, y) d y, \quad x \in \mathbb{R}^{N} \\
& =\lim _{\varepsilon \rightarrow 0} \int_{\mathbb{R}^{N} \backslash B_{\varepsilon}(x)}|u(x)-u(y)|^{p(x, y)-2}(u(x)-u(y)) K(x, y) d y, \quad x \in \mathbb{R}^{N},
\end{aligned}
$$

where $p: \mathbb{R}^{N} \times \mathbb{R}^{N} \longrightarrow(1,+\infty)$ is a continuous bounded function satifying (1.2), (1.3) and

$$
p((x, y)-(z, z))=p(x, y) \quad \forall(x, y),(z, z) \in \mathbb{R}^{N} \times \mathbb{R}^{N},
$$

The kernel $K: \mathbb{R}^{N} \times \mathbb{R}^{N} \longrightarrow(0,+\infty)$ is a measurable function with the following properties:

$$
K(x, y)=K(y, x) \quad \forall(x, y) \in \mathbb{R}^{N} \times \mathbb{R}^{N}
$$

and $\exists k_{0}>0$ such that

$$
\begin{aligned}
& K(x, y) \geq k_{0}|x-y|^{-(N+s p(x, y))} \quad(x, y) \in \mathbb{R}^{N} \times \mathbb{R}^{N} \quad \text { with } \quad x \neq y \\
& g K \in L^{1}\left(\mathbb{R}^{N} \times \mathbb{R}^{N}\right), \quad \text { where } \quad g(x, y)=\min \left\{1,|x-y|^{p(x, y)}\right\} .
\end{aligned}
$$

A typical example for $K$ is given by the singular kernel $K(x, y)=|x-y|^{-(N+s p(x, y))}$, in this case $\mathcal{L}_{K}^{p(x)}=\left(-\Delta_{p}\right)^{s}$.

We will introduce the functional space which was introduced in [2] by Benkirane et al., we give the general fractional Sobolev space with variable exponent as follows

$X=W^{K, p(x, y)}(\Omega)=\left\{\begin{array}{l}u: \mathbb{R}^{N} \longrightarrow \mathbb{R} \text { measurable, such that } u \in L^{\bar{p}(x)} \text { with } \\ \int_{Q} \frac{|u(x)-u(y)|^{p(x, y)}}{\lambda^{p(x, y)}} K(x, y) d x d y<+\infty, \quad \text { for some } \lambda>0\end{array}\right\}$,

where $\Omega$ be an open bounded subset of $\mathbb{R}^{N}$ and $Q$ defined by

$$
Q:=\mathbb{R}^{N} \times \mathbb{R}^{N} \backslash(\mathcal{C} \Omega \times \mathcal{C} \Omega), \quad \text { with } \quad \mathcal{C} \Omega=\mathbb{R}^{N} \backslash \Omega .
$$

Note that, the space $W^{K, p(x, y)}(\Omega)$ is a Banach space (see [2] ) and endowed with the norm

$$
\|u\|_{W^{K, p(x, y)}(\Omega)}=\|u\|_{K, p(x, y)}=\|u\|_{L^{p(x)}(\Omega)}+[u]_{K, p(x, y)}
$$

where,

$$
[u]_{K, p(x, y)}=\inf \left\{\lambda>0: \int_{Q} \frac{|u(x)-u(y)|^{p(x, y)}}{\lambda^{p(x, y)}} K(x, y) d x d y \leq 1\right\} .
$$

The space $\left(X,\|\cdot\|_{X}\right)$ is separable and uniformly convex reflexive, see [2].

In this paper, we are inspired by the results on the $p(x)$-Laplacian problems with weight introduced in $[1,5,18]$ and some results on the theory of fractional Sobolev spaces with variable exponent due to Kaufmann et al. [17] and Bahrouni et al. [4]. The aim of this paper is to investigate problem (1.1) by adapting the variational techniques. We will study a class of eigenvalue problems with indefinite weight for fractional $p(x, y)$-Laplacian equations and we establish that any $\lambda>0$ suficiently small is an eigenvalue of the above nonhomogeneous nonlocal problem. The proof relies on some variational arguments based on Ekeland's variational principle.

The main result of the present paper reads as follows: 
Theorem 1.1. Let $\Omega$ be a smooth bounded domain in $\mathbb{R}^{N}$ and let $s \in(0,1)$. Suppose that

$\left(H_{1}\right) p: \mathbb{R}^{N} \times \mathbb{R}^{N} \longrightarrow(1,+\infty)$ be a continuous variable exponent with $\operatorname{sp}(x, y)<N$ for all $(x, y) \in \mathbb{R}^{N} \times \mathbb{R}^{N}$ satisfying (1.2) and (1.3).

$\left(H_{2}\right) q: \bar{\Omega} \longleftarrow(1,+\infty)$ be a continuous bounded variable exponent satisfy (1.4).

$\left(H_{3}\right) K: \mathbb{R}^{N} \times \mathbb{R}^{N}(0,+\infty)$ is a measurable function satisfying (1.12), (1.13) and (1.14).

$\left(H_{4}\right) V \in L^{\sigma(x)}(\Omega)$ and there exists a measurable set $\Omega_{0} \subset \subset \Omega$ of positive measure such that $V(x)>0$ for all $x \in \Omega_{0}$, where $\sigma: \bar{\Omega} \longrightarrow \mathbb{R}$.

$\left(H_{5}\right) 1<q(x)<p^{-} \leqslant p^{+}<\frac{N}{s}<\sigma(x)$ for all $x \in \bar{\Omega}$.

Then there exists $\lambda^{\star}>0$ such that for all $\lambda \in\left(0, \lambda^{\star}\right)$ is an eigenvalue of problem $\left(\mathcal{P}_{K}\right)$.

The rest of this paper is structured as follows. Section 2 states some preliminary properties to establish our results presented in Section 3. In section 3, we establish and prove our main theorem.

\section{Preliminaries and technical Lemmas}

In this section, we recall some definitions and some properties about generalized Lebesgue spaces $L^{r(x)}(\Omega)$ and fractional Sobolev spaces with variable exponent, which we will use later (For more details see [2, 13, 14, 19]).

Define the generalized Lebesgue space by:

$$
L^{r(x)}(\Omega)=\left\{u: \quad \Omega \longrightarrow \mathbb{R}, \quad \text { measurable and } \quad \int_{\Omega}|u(x)|^{p(x)} d x<\infty\right\}
$$

where

$$
r \in C_{+}(\bar{\Omega}) \quad \text { and } \quad C_{+}(\bar{\Omega})=\{r \in C(\bar{\Omega}): r(x)>1, \quad \forall x \in \bar{\Omega}\} .
$$

Denote $r^{+}=\max _{x \in \bar{\Omega}} r(x)$ and $r^{-}=\min _{x \in \bar{\Omega}} r(x)$, such that

$$
1<r^{-} \leq r(x) \leq r^{+}<+\infty .
$$

The space $L^{r(x)}(\Omega)$ endowed with the Luxemburg norm

$$
|u|_{r(x)}=\inf \left\{\mu>0 ; \quad \int_{\Omega}\left|\frac{u(x)}{\mu}\right|^{r(x)} d x \leq 1\right\}
$$

and the space $\left(L^{r(x)}(\Omega),|\cdot|_{r(x)}\right)$ is a Banach.

Proposition 2.1. ([14]) The space $\left(L^{r(x)}(\Omega),|\cdot|_{r(x)}\right)$ is separable, uniformly convex, reflexive and its conjugate space is $L^{\hat{r}(x)}(\Omega)$ where $\hat{r}(x)$ is the conjugate function of $r(x)$ i.e

$$
\frac{1}{r(x)}+\frac{1}{\hat{r}(x)}=1, \quad \forall x \in \Omega \text {. }
$$

For all $u \in L^{r(x)}(\Omega)$ and $v \in L^{\hat{r}(x)}(\Omega)$ the Hölder's type inequality

$$
\left|\int_{\Omega} u v d x\right| \leq\left(\frac{1}{r^{-}}+\frac{1}{\hat{r}^{-}}\right)|u|_{r(x)}|v|_{\hat{r}(x)}
$$

holds true.

Moreover, if $r_{1}, r_{2}, r_{3} \in C_{+}(\bar{\Omega})$ and $\frac{1}{r_{1}(x)}+\frac{1}{r_{2}(x)}+\frac{1}{r_{3}(x)}=1$, then for any $u \in L^{r_{1}(x)}(\Omega)$, $v \in L^{r_{2}(x)}(\Omega)$ and $w \in L^{r_{3}(x)}(\Omega)$ the following inequality holds (see [14],proposition 2.5):

$$
\int_{\Omega}|u v w| d x \leqslant\left(\frac{1}{r_{1}^{-}}+\frac{1}{r_{2}^{-}}+\frac{1}{r_{3}^{-}}\right)|u|_{r_{1}(x)}|v|_{r_{2}(x)}|w|_{r_{3}(x)} .
$$


Furthermore, if we define the mapping $\rho: L^{r(x)}(\Omega) \rightarrow \mathbb{R}$ by

$$
\rho_{r(x)}(u)=\int_{\Omega}|u|^{r(x)} d x
$$

we have the following proposition:

Proposition 2.2. ([14])

$$
\begin{gathered}
|u|_{r(x)}<1(=1,>1) \Leftrightarrow \rho_{r(x)}(u)<1(=1,>1) \\
|u|_{r(x)}>1 \Rightarrow|u|_{r(x)}^{r-} \leqslant \rho(u)_{r(x)} \leqslant|u|_{r(x)}^{r+} \\
|u|_{r(x)}<1 \Rightarrow|u|_{r(x)}^{r+} \leqslant \rho_{r(x)}(u) \leqslant|u|_{r(x)}^{r-} \\
\left|u_{n}-u\right|_{r(x)} \longrightarrow 0 \Leftrightarrow \rho_{r(x)}\left(u_{n}-u\right) \longrightarrow 0
\end{gathered}
$$

We recall the following proposition, which will be needed later:

Proposition 2.3. ([12]) Let $r_{1}$ and $r_{2}$ be measurable functions such that $r_{1} \in L^{\infty}(\Omega)$ and $1<r_{1}(x) r_{2}(x) \leqslant \infty$, for a.e. $x \in \Omega$. Let $u \in L^{r_{2}(x)}(\Omega), u \neq 0$. Then

$$
\begin{gathered}
|u|_{r_{1}(x) r_{2}(x)} \leqslant 1 \Rightarrow|u|_{r_{1}(x) r_{2}(x)}^{r_{1}^{+}} \leqslant\left.\left.|| u\right|^{r_{1}(x)}\right|_{r_{2}(x)} \leqslant|u|_{r_{1}(x) r_{2}(x)}^{r_{1}^{-}} . \\
|u|_{r_{1}(x) r_{2}(x)} \geqslant 1 \Rightarrow|u|_{r_{1}(x) r_{2}(x)}^{r_{1}^{-}} \leqslant\left.\left.|| u\right|^{r_{1}(x)}\right|_{r_{2}(x)} \leqslant|u|_{r_{1}(x) r_{2}(x)}^{r_{1}^{+}} .
\end{gathered}
$$

Theorem 2.4. ([2]) Let $\Omega$ be a smooth bounded domain in $\mathbb{R}^{N}$ and let $s \in(0,1)$. Let $p: \mathbb{R}^{N} \times \mathbb{R}^{N} \longrightarrow(1,+\infty)$ be a continuous variable exponent with $s p(x, y)<N$ for all $(x, y) \in \mathbb{R}^{N} \times \mathbb{R}^{N}$. Let (1.2) and (1.3) be satisfied and $q: \bar{\Omega} \longrightarrow(1,+\infty)$ be a continuous bounded variable exponent such that,

$$
1<r(x)<p_{s}^{\star}=\frac{N \bar{p}(x)}{N-\bar{p}(x)}, \quad \forall x \in \bar{\Omega} .
$$

Suppose that $K: \mathbb{R}^{N} \times \mathbb{R}^{N} \longrightarrow(0,+\infty)$ is a measurable function satisfying (1.12), (1.13) and (1.14). Then

(i) There exists a positive constant $C=C(N, p, q, s, \Omega)>0$, such that for any $u \in W^{K, p(x, y)}(\Omega)$, we have

$$
\|u\|_{q(x)} \leq C\|u\|_{s, p(x, y)} \leq C \max \left\{1, \tilde{k}_{0}\right\}\|u\|_{K, p(x, y)}
$$

where $\tilde{k}_{0}=\tilde{k}_{0}\left(k_{0}, p^{-}, p^{+}\right)$is a positive constant. That is, the space $W^{K, p(x, y)}(\Omega)$ is continuously embedded in $L^{q(x)}(\Omega)$. Moreover, this embedding is compact.

(ii) There exists a positive constant $C_{0}=C_{0}\left(N, p, s, \tilde{k}_{0}, \Omega\right)>0$, such that

$$
[u]_{K, p(x, y)} \leq\|u\|_{K, p(x, y)} \leq C_{0}[u]_{K, p(x, y)} .
$$

For any $u \in W^{K, p(x, y)}$, we define the modular $\rho_{K, p(. .,)}$ by

$$
\rho_{K, p(., .)}(u)=\int_{Q}|u(x)-u(y)|^{p(x, y)} K(x, y) d x d y+\int_{\Omega}|u(x)|^{\bar{p}(x)} d x
$$

and it is convex on $W^{K, p(x, y)}$. The norme associated with $\rho_{K, p(., .)}$ is given by

$$
\|u\|_{\rho_{K}, p(., .)}=\inf \left\{\lambda>0: \rho_{K, p(., .)}\left(\frac{u}{\lambda}\right) \leq 1\right\} .
$$

Remark 2.5. $\rho_{K, p(., .)}$ also check the results of Proposition 2.2.

Using the same argument as in $([11]$, Theorem 2.17$)$, we prove that $\|\cdot\|_{\rho_{K}, p(. .,)}$ is a norm on $W^{K, p(x, y)}(\Omega)$, which is equivalent to the norm $\|\cdot\|_{K, p(., .)}$. We also define the closed linear subspace of $W^{K, p(x, y)}(\Omega)$ by

$$
X_{0}=W_{0}^{K, p(x, y)}(\Omega)=\left\{u \in W^{K, p(x, y)}(\Omega): u(x)=0 \quad \text { a.e. } \quad \text { in } \quad \mathbb{R}^{N} \backslash \Omega\right\} .
$$


Remark 2.6. (i) The assertion (i) in Theorem 2.1 implies that $W^{K, p(x, y)}(\Omega)$ and $W_{0}^{K, p(x, y)}(\Omega)$ are continuously embedded in $L^{q(x)}(\Omega)$, where $1<q(x)<p_{s}^{\star}(x)$ for all $x \in \Omega$.

(ii) As a consequence of Theorem 2.4. $1-(i i),[\cdot]_{K, p(x, y)}$ is an equivalent norm of $\|u\|_{K, p(x, y)}$ on $W_{0}^{K, p(x, y)}(\Omega)$.

(iii) $\left(W_{0}^{K, p(x, y)}(\Omega),[.]_{K, p(x ; y)}\right)$ is a separable, reflexive, and uniformly convex Banach space (see [2], Lemma 3.5).

On the other hand, for any $u \in W_{0}^{K, p(x, y)}(\Omega)$, we define the functional

$$
\rho_{K, p(. .)}^{o}(u)=\int_{Q}|u(x)-u(y)|^{p(x, y)} K(x, y) d x d y .
$$

$\rho_{K, p(. .,)}^{o}$ is a convexe modular on $W_{0}^{K, p(x, y)}(\Omega)$. The norm associated with $\rho_{K, p(., .)}^{o}$ is given by

$$
\|u\|_{\rho_{K, p(\ldots)}^{o}}=[u]_{K, p(x, y)}=\inf \left\{\lambda>0: \rho_{K, p(., .)}^{o}\left(\frac{u}{\lambda}\right) \leq 1\right\}=\|u\|_{X_{0}} .
$$

Remark 2.7. $\quad \rho_{K, p(\ldots)}^{o}$ also check the results of Proposition 2.2.

We recall also the following properties:

Lemma 2.8. ([2]) Let $p: \mathbb{R} \times \mathbb{R} \longrightarrow(1,+\infty)$ be a continuous variable exponent and $K: \mathbb{R} \times \mathbb{R} \longrightarrow(0,+\infty)$ is a measurable function satisfy (1.12) and (1.14). Then For any $u \in W_{0}^{K, p(x, y)}$, we have

(i) $1 \leq[u]_{K, p(x, y)} \Rightarrow[u]_{K, p(x, y)}^{p^{-}} \leq \rho_{K, p(\ldots)}^{o}(u) \leq[u]_{K, p(x, y)}^{p^{+}}$.

(ii) $[u]_{K, p(x, y)} \leq 1 \Rightarrow[u]_{K, p(x, y)}^{p^{+}} \leq \rho_{K, p(\cdot, \cdot)}^{o}(u) \leq[u]_{K, p(x, y)}^{p^{-}}$.

Lemma 2.9. ([2]) Let (1.2), (1.3) and (1.11) be satisfied. Then the space $C_{0}^{\infty}\left(\mathbb{R}^{N}\right)$ of smooth functions with compact support is dense in $W^{K, p(x, y)}(\Omega)$.

Let (1.2) and (1.3), be satisfied and let $K: \mathbb{R} \times \mathbb{R} \longrightarrow(0,+\infty)$ is a measurable function satisfy (1.12), (1.13) and (1.14). Then

$$
\begin{aligned}
\mathcal{L}_{K}^{p(x)}: X_{0} & \longrightarrow X_{0}^{\star} \\
u \mapsto \mathcal{L}_{K}^{p(x)}(u): X_{0} & \longrightarrow \mathbb{R} \\
\varphi & \mapsto<\mathcal{L}_{K}^{p(x)}(u), \varphi>
\end{aligned}
$$

such that

$$
<\mathcal{L}_{K}^{p(x)}(u), \varphi>=\int_{\mathbb{R}^{2 N}}|u(x)-u(y)|^{p(x, y)-2}(u(x)-u(y))(\varphi(x)-\varphi(y)) K(x, y) d x d y .
$$

Where $X_{0}^{\star}=\left(W_{0}^{K, p(x, y)}(\Omega)\right)^{\star}$ is the the dual of $X_{0}=W_{0}^{K, p(x, y)}(\Omega)$.

In the following Lemma, we introduce fundamental properties of the operator $\mathcal{L}_{K}^{p(x)}$.

Lemma 2.10. ([2]) Suppose that (1.2) and (1.3) be satisfied and let $K: \mathbb{R} \times \mathbb{R} \longrightarrow$ $(0,+\infty)$ be a measurable function which satisfies (1.12), (1.13) and (1.14). Then, The following assertions hold:

(i) $\mathcal{L}_{K}^{p(x)}$ is well defined and bounded.

(ii) $\mathcal{L}_{K}^{p(x)}$ is a strictly monotone operator. 
(iii) $\mathcal{L}_{K}^{p(x)}$ is mapping of type $\left(S_{+}\right)$, that is,

$\left\{\begin{array}{l}u_{k} \rightarrow u \quad \text { in } \quad X_{0} \\ \lim _{k \rightarrow+\infty} \sup <\mathcal{L}_{K}^{p(x)}(u)_{k}-\mathcal{L}_{K}^{p(x)}(u), u_{k}-u>\leq 0, \quad \Rightarrow u_{k} \longrightarrow u \quad \text { in } \quad W_{0}^{K, p(x, y)}(\Omega) .\end{array}\right.$

(iv) $\mathcal{L}_{K}^{p(x)}$ is a homeomorphisme.

(v) $\mathcal{L}_{K}^{p(x)}$ is coercive.

\section{MAin RESUlt}

We begin by the following definition.

Definition 3.1. We say that $u \in X_{0}$ is a weak solution of problem $\left(\mathcal{P}_{K}\right)$, if for all $\varphi \in X_{0}$, we have

$$
\begin{gathered}
\int_{Q}|u(x)-u(y)|^{p(x, y)-2}(u(x)-u(y))(\varphi(x)-\varphi(y)) K(x, y) d x d y \\
+\int_{\Omega}|u(x)|^{\bar{p}(x)-2} u(x) \varphi(x) d x-\lambda \int_{\Omega} V(x)|u(x)|^{q(x)-2} u(x) \varphi(x) d x=0 .
\end{gathered}
$$

Moreover, we say that $\lambda$ is an eigenvalue of problem $\left(\mathcal{P}_{K}\right)$, if there exists $u \in X_{0} \backslash\{0\}$ which satisfies (3.18), i.e. $u$ is the corresponding eigenfunction to $\lambda$.

Let us consider the functional $I_{\lambda}: X_{0} \longrightarrow \mathbb{R}$ associated with problem $\left(\mathcal{P}_{K}\right)$ by

$$
I_{\lambda}(u)=J(u)+\Phi(u)-\lambda \Psi(u)
$$

where

$$
J(u)=\int_{Q} \frac{1}{p(x, y)}|u(x)-u(y)|^{p(x, y)} K(x, y) d x d y, \quad \Phi(u)=\int_{\Omega} \frac{1}{\bar{p}(x)}|u(x)|^{\bar{p}(x)} d x
$$

and

$$
\Psi(u)=\int_{\Omega} \frac{1}{q(x)} V(x)|u(x)|^{q(x)} d x
$$

for any $\lambda>0$.

We use the same arguments as in $([3,23])$, we show the following lemma:

Lemma 3.2. Let $\Omega$ be a smooth bounded domain in $\mathbb{R}^{N}$ and let $s \in(0,1)$. Let $p$ : $\mathbb{R}^{N} \times \mathbb{R}^{N} \longrightarrow(1,+\infty)$ be a continuous variable exponent with $\operatorname{sp}(x, y)<N$ for all $(x, y) \in \mathbb{R}^{N} \times \mathbb{R}^{N}$. Let (1.2) and (1.3) be satisfied. Let $q: \bar{\Omega} \longrightarrow(1,+\infty)$ be a continuous bounded variable exponent satisfy (1.4). Suppose that $K: \mathbb{R}^{N} \times \mathbb{R}^{N} \longrightarrow(0,+\infty)$ is a mesurable function satisfying (1.13) and (1.14). Then:

(1) $I_{\lambda}$ is well defined.

(2) $I_{\lambda} \in\left(W_{0}^{K, p(x, y)}(\Omega), \mathbb{R}\right)$ and for all $u, \varphi \in W_{0}^{K, p(x, y)}(\Omega)$, its Gâteaux derivative is given by:

$$
\begin{aligned}
\left\langle I_{\lambda}^{\prime}(u), \varphi\right\rangle & =\int_{Q}|u(x)-u(y)|^{p(x, y)-2}(u(x)-u(y))(\varphi(x)-\varphi(y)) K(x, y) d x d y \\
& +\int_{\Omega}|u(x)|^{\bar{p}(x)-2} u(x) \varphi(x) d x-\lambda \int_{\Omega} V(x)|u(x)|^{q(x)-2} u(x) \varphi(x) d x .
\end{aligned}
$$

The following result shows that the functional $I_{\lambda}$ satisfies the first geometrical condition of the mountain pass theorem. 
Lemma 3.3. Let be $\Omega$ a smooth bounded domain in $\mathbb{R}^{N}$ and let $s \in(0,1)$. Let $p$ : $\mathbb{R}^{N} \times \mathbb{R}^{N} \longrightarrow(1,+\infty)$ be a continuous variable exponent with $s p(x, y)<N$ for all $(x, y) \in \mathbb{R}^{N} \times \mathbb{R}^{N}$. Let (1.2) and (1.3) be satisfied. Let $q: \bar{\Omega} \longrightarrow(1,+\infty)$ be a continuous bounded variable exponent satisfy (1.4). Suppose that $K: \mathbb{R}^{N} \times \mathbb{R}^{N} \longrightarrow(0,+\infty)$ is a measurable function satisfying (1.12), (1.13) and (1.14). Then, there exists $\lambda^{\star}>0$ such that for any $\lambda \in\left(0, \lambda^{\star}\right)$, there exist $R, a>0$ such that $I_{\lambda}(u)>a>0$ for any $u \in X_{0}$ with $\|u\|_{X_{0}}=R$.

Proof of Lemma 3.3: Using the same argument as in ([23], see Theorem 3.2).

Indeed, since $\alpha(x)=\frac{\sigma(x) q(x)}{\sigma(x)-1}<p_{s}^{\star}(x)$ for all $x \in \Omega$, the embedding $X_{0} \hookrightarrow L^{\alpha(x)}(\Omega)$ is continuous, there exists $c_{1}>0$ such that

$$
\|u\|_{\alpha(x)} \leq c_{1}\|u\|_{X_{0}}, \forall u \in X_{0} .
$$

From (3.20), for any $u \in X_{0}$ with $\|u\|=R$ small enough, we have

$$
\begin{aligned}
I_{\lambda}(u) & \geq \frac{1}{p^{+}} \int_{Q}|u(x)-u(y)|^{p(x, y)} K(x, y) d x d y+\frac{1}{p^{+}} \int_{\Omega}|u(x)|^{\bar{p}(x)} d x \\
& -\frac{\lambda}{q^{-}} \int_{\Omega} V(x)|u(x)|^{q(x)} d x \\
& \geq \frac{1}{p^{+}}\left[\int_{Q}|u(x)-u(y)|^{p(x, y)} K(x, y) d x d y+\int_{\Omega}|u(x)|^{\bar{p}(x)} d x\right] \\
& -\frac{\lambda}{q^{-}} \int_{\Omega} V(x)|u(x)|^{q(x)} d x \\
& \geq \frac{1}{p^{+}}\|u\|_{X_{0}}^{p+}-\frac{2 \lambda}{q^{-}} c_{1}^{q^{-}}|V|_{\sigma(x)}\|u\|_{X_{0}}^{q^{-}} \\
& \geq \frac{1}{p^{+}} R^{p^{+}}-\frac{2 \lambda}{q^{-}} c_{1}^{q^{-}}|V|_{\sigma(x)} R^{q^{-}} \\
& \geq R^{q^{-}}\left(\frac{1}{p^{+}} R^{p^{+}-q^{-}}-\frac{2 \lambda}{q^{-}} c_{1}^{q^{-}}|V|_{\sigma(x)}\right) .
\end{aligned}
$$

Defining

$$
\lambda^{\star}=\frac{R^{p^{+}-q^{-}}}{2 p^{+}} \cdot \frac{q^{-}}{2 c_{1}|V|_{\sigma(x)}},
$$

we can conclude that for any $\lambda \in\left(0, \lambda^{\star}\right)$ and any $u \in X_{0}$ with $\|u\|_{X_{0}}=R$, there exists $a=\frac{R^{p+}}{2 p^{+}}>0$ such that

this completes the proof of Lemma 3.3.

$$
I_{\lambda} \geq a>0
$$

The following result shows that the functional $I_{\lambda}$ satisfies the second geometrical condition of mountain pass theorem.

Lemma 3.4. Let $\Omega$ be a smooth bounded domain in $\mathbb{R}^{N}$ and let $s \in(0,1)$. Let $p: \mathbb{R} \times$ $\mathbb{R} \longrightarrow(1,+\infty)$ be a continuous variable exponent with $\operatorname{sp}(x, y)<N$ for all $(x, y) \in \mathbb{R} \times \mathbb{R}$. Let (1.2) and (1.3) be satisfied. Let $q: \bar{\Omega} \longrightarrow(1,+\infty)$ be a continuous bounded variable exponent satisfy (1.4). Suppose that $K: \mathbb{R} \times \mathbb{R} \longrightarrow(0,+\infty)$ is a measurable function satisfying (1.12), (1.13) and (1.14).

Then, there exists $\varphi \in X_{0}$ such that $\varphi \geq 0, \varphi \neq 0$ and $I_{\lambda}(t \varphi)<0$ for any $t$ small enough.

Proof of Lemma 3.4: Using the same argument as in ([23], see Theorem 3.3).

Assumption (1.14) implies that $q^{-}<p^{-}$. Let $\varepsilon>0$ be such that $q^{-}+\varepsilon \leq p^{-}$since $q \in C(\bar{\Omega})$, then we can find an open set $\Omega_{0} \subset \Omega$ such that

$$
\left|q(x)-q^{-}\right| \leq \varepsilon \quad \forall x \in \Omega_{0}
$$


Consequently

$$
q(x) \leq q^{-}+\varepsilon \leq p^{-} \quad \forall x \in \Omega_{0} .
$$

Let $\varphi \in C_{0}^{\infty}(\Omega)$ be such that $\bar{\Omega}_{0} \subset \operatorname{supp}(\varphi), \varphi(x)=1$ for all $x \in \Omega_{0}$, and $0 \leq \varphi \leq 1$ in $\Omega$. Then for any $t \in(0,1)$ we have

$$
\begin{aligned}
I_{\lambda}(t \varphi) & =\int_{Q} \frac{t^{p(x, y)}}{p(x, y)}|\varphi(x)-\varphi(y)|^{p(x, y)} K(x, y) d x d y+\int_{\Omega} \frac{t^{\bar{p}(x)}}{\bar{p}(x)}|\varphi(x)|^{\bar{p}(x)} d x \\
& -\lambda \int_{\Omega} \frac{t^{q(x)}}{q(x)} V(x)|\varphi(x)|^{q(x)} d x \\
& \leq \frac{t^{p^{-}}}{p^{-}}\left[\int_{Q}|\varphi(x)-\varphi(y)|^{p(x, y)} K(x, y) d x d y+\int_{\Omega}|\varphi(x)|^{\bar{p}(x)} d x\right] \\
& -\lambda \int_{\Omega_{0}} \frac{t^{q(x)}}{q(x)} V(x)|\varphi(x)|^{q(x)} d x \\
& \leq \frac{t^{p^{-}}}{p^{-}} \rho_{K, p(. . .)}(u)-\frac{\lambda}{q^{+}} t^{q^{-}+\varepsilon} \int_{\Omega_{0}} V(x)|\varphi(x)|^{q(x)} d x \\
& \leq t^{q^{-}+\varepsilon}\left[\frac{\rho_{K, p(.,)}(\varphi)}{p^{-}} t^{p^{-}-q^{-}-\varepsilon}-\frac{\lambda}{q^{+}} \int_{\Omega_{0}} V(x)|\varphi(x)|^{q(x)} d x\right] .
\end{aligned}
$$

Therefore

$$
I_{\lambda(t \varphi)}<0 \quad \text { for any } \quad t<\xi^{\frac{1}{p^{-}-q^{-}-\varepsilon}}
$$

where

$$
0<\xi<\min \left\{1, \frac{\frac{\lambda}{q^{+}} \int_{\Omega_{0}} V(x)|\varphi(x)|^{q(x)} d x}{\rho_{K, p(., .)}(\varphi)}\right\} .
$$

Finally, we point out that $\rho_{p(. .)}(\varphi)>0$ (this fact implies that $\varphi \neq 0$ ). Indeed, since $\bar{\Omega}_{0} \subset \operatorname{supp}(\varphi) \subset \Omega$, and $0 \leq \varphi \leq 1$ in $\Omega$, so we get

$$
0<\int_{\Omega_{0}}|\varphi(x)|^{q(x)} d x \leq \int_{\Omega}|\varphi(x)|^{q(x)} d x \leq \int_{\Omega}|\varphi(x)|^{q^{-}} d x .
$$

On the other hand, since $1<q^{-}<p_{s}^{\star}(x)$ for all $x \in \bar{\Omega}$, then $X_{0}$ is continuously embedded in $L^{q^{-}}(\Omega)$, so there exists $c_{2}>0$ such that

$$
\|\varphi\|_{L^{q^{-}}(\Omega)} \leq c_{2}\|\varphi\|_{X_{0}} .
$$

Combining (3.22) and (3.23), we get

$$
0<\frac{1}{c_{2}}\|\varphi\|_{L^{q^{-}}(\Omega)} \leq\|\varphi\|_{X_{0}}
$$

Using the last relation and Proposition 2.2, we deduce that

$$
\rho_{p(. . .)}(\varphi)>0
$$

and the conclusion is completed.

Proof of Theorem 1.1:

Let $\lambda^{\star}$ be defined as in (3.21) and let $\lambda \in\left(0, \lambda^{\star}\right)$. By Lemma 3.3, it follows that

$$
\inf _{\partial B_{R}(0)} I_{\lambda}>0
$$

where $\partial B_{R}(0)=\left\{u \in \partial B_{R}(0):\|u\|_{X_{0}}=R\right\}$ and $\partial B_{R}(0)$ is the ball centered at the origin and of radius $R$ in $X_{0}$.

On the other hand, by Lemma 3.4, there exists $\varphi \in X_{0}$ such that $I_{\lambda}(t \varphi)<0$ for any $t$ 
small enough.

Moreover, for all $u \in B_{R}(0)$, we have

$$
I_{\lambda}(u) \geq \frac{1}{p^{+}}\|u\|_{X_{0}}^{p^{+}}-\frac{\lambda}{r^{-}} c_{1}^{q^{-}}|V|_{\sigma(x)}\|u\|_{X_{0}}^{q^{-}} .
$$

Then we have

$$
-\infty<\bar{c}=\inf _{u \in \bar{B}_{R}(0)} I_{\lambda}(u)<0 .
$$

Combining (3.22) and (3.24), then we can assume that

$$
0<\varepsilon<\inf _{\partial B_{R}(0)} I_{\lambda}-\inf _{B_{R}(0)} I_{\lambda} .
$$

Then, by applying Ekeland's variational principale $([16])$ to the functional $I_{\lambda}: \bar{B}_{R}(0) \longrightarrow$ $\mathbb{R}$, there exists $u_{\varepsilon} \in \bar{B}_{R}(0)$ such that

$$
\left\{\begin{aligned}
I_{\lambda}\left(u_{\varepsilon}\right) & <\inf _{u \in \bar{B}_{R}(0)} I_{\lambda}(u)+\varepsilon \\
I_{\lambda}\left(u_{\varepsilon}\right) & <I_{\lambda}(u)+\varepsilon\left\|u-u_{\varepsilon}\right\|_{X_{0}}, \forall u \neq u_{\varepsilon} .
\end{aligned}\right.
$$

So,

$$
I_{\lambda}\left(u_{\varepsilon}\right)<\inf _{u \in \partial B_{R}(0)} I_{\lambda}(u)
$$

It follows that $u_{\varepsilon} \in B_{R}(0)$.

Now, we consider

$$
\begin{aligned}
& I_{\lambda}^{\varepsilon}: \bar{B}_{R}(0) \longrightarrow \mathbb{R} \\
& \quad u \longrightarrow I_{\lambda}(u)+\varepsilon\left\|u-u_{\varepsilon}\right\|_{X_{0}} .
\end{aligned}
$$

By (3.23), we get

$$
I_{\lambda}^{\varepsilon}\left(u_{\varepsilon}\right)=I_{\lambda}(u)<I_{\lambda}^{\varepsilon}(u), \forall u \neq u_{\varepsilon} .
$$

Thus $u_{\varepsilon}$ is a minimum point of $I_{\lambda}^{\varepsilon}$ on $\bar{B}_{R}(0)$. It follows that for any $t>0$ small enough and $v \in B_{R}(0)$

$$
\frac{I_{\lambda}^{\varepsilon}\left(u_{\varepsilon}+t v\right)-I_{\lambda}^{\varepsilon}\left(u_{\varepsilon}\right)}{t} \geq 0 .
$$

By this fact, we claim that

$$
\frac{I_{\lambda}\left(u_{\varepsilon}+t v\right)-I_{\lambda}\left(u_{\varepsilon}\right)}{t}+\varepsilon\|v\|_{X_{0}} \geq 0
$$

When $\mathrm{t}$ tends to $0^{+}$, we get

$$
<I_{\lambda}^{\prime}\left(u_{\varepsilon}\right), v>+\varepsilon\|v\|_{X_{0}} \geq 0 .
$$

This implies that

$$
\left\|I_{\lambda}\left(u_{\varepsilon}\right)\right\|_{X_{0}^{\star}} \leq \varepsilon
$$

From ( 3.28$)$, we deduce that there exists a sequence $\left(w_{n}\right) \subset B_{R}(0)$ such that

$$
I_{\lambda}\left(w_{n}\right) \longrightarrow \bar{c} \quad \text { and } \quad I_{\lambda}^{\prime}\left(w_{n}\right) \longrightarrow 0 .
$$

By the relations (3.25) and (3.29), we have that $\left(w_{n}\right)$ is bounded in $X_{0}$. Thus there exists $w \in X_{0}$ such that $w_{n} \rightarrow w$ in $X_{0}$.

By (1.4), we have that $q(x)<p_{s}^{\star}(x)$ for all $x \in \bar{\Omega}$, so by Theorem 2.4 and Remark 2.6 we deduce that $X_{0}$ is compactly embedded in $L^{q(x)}(\Omega)$, then

$$
w_{n} \longrightarrow w \quad \text { in } \quad L^{q(x)}(\Omega) .
$$


and

$$
\begin{aligned}
\left.\left|\int_{\Omega} V(x)\right| w_{n}\right|^{q(x)-2} w_{n}\left(w_{n}-w\right) d x \mid & \leq\left.\left. 3|V|_{\sigma(x)}|| w_{n}\right|^{q(x)-2} w_{n}\right|_{\frac{q(x)}{(q(x)-1)}}\left|w_{n}-w\right|_{\beta(x)} \\
& \leq 3|V|_{\sigma(x)}\left(1+\left|w_{n}\right|_{q(x)}^{q^{+}}-1\right)\left|w_{n}-w\right|_{\beta(x)} \\
& \rightarrow 0 \quad \text { as } n \rightarrow \infty,
\end{aligned}
$$

where $\beta(x)=\frac{\sigma(x) q(x)}{(\sigma(x)-q(x))}$.

By the same argument, we have

$$
\lim _{n \rightarrow+\infty} \int_{\Omega}\left|w_{n}\right|^{\bar{p}(x)-2} w_{n}\left(w_{n}-w\right) d x=0 .
$$

According to ( 3.29 ), we conclude that

$$
\lim _{n \rightarrow+\infty}<I_{\lambda}^{\prime}\left(w_{n}\right), w_{n}>=0
$$

Namely,

$$
\begin{aligned}
& \int_{\Omega \times \Omega}\left|w_{n}(x)-w_{n}(y)\right|^{p(x, y)-2}\left(w_{n}(x)-w_{n}(y)\right)\left(\left(w_{n}(x)-w(x)\right)-\left(w_{n}(y)-w(y)\right)\right) \\
& \quad \times K(x, y) d x d y+\int_{\Omega}\left|w_{n}\right|^{\bar{p}(x)-2} w_{n}\left(w_{n}-w\right) d x \\
& \quad-\lambda \int_{\Omega} V(x)\left|w_{n}\right|^{q(x)-2} w_{n}\left(w_{n}-w\right) d x \rightarrow 0 \quad \text { as } n \rightarrow \infty
\end{aligned}
$$

And so,

$$
\begin{array}{r}
\lim _{n \rightarrow+\infty} \int_{\Omega \times \Omega}\left|w_{n}(x)-w_{n}(y)\right|^{p(x, y)-2}\left(w_{n}(x)-w_{n}(y)\right)\left(\left(w_{n}(x)-w_{n}(y)\right)\right. \\
-(w(x)-w(y))) \times K(x, y) d x d y=0 .
\end{array}
$$

Consequently, using Lemma 2.10 ( ii), and the fact that $w_{n} \rightarrow w$ in $X_{0}$, we get

$$
\left\{\begin{array}{l}
\lim \sup _{n}<\mathcal{L}\left(w_{n}\right), w_{n}-w>\leq 0 \\
w_{n} \rightarrow w \text { in } X_{0}, \\
\mathcal{L} \text { is a mapping of type } \quad\left(S_{+}\right)
\end{array} \quad \Rightarrow w_{n} \longrightarrow w \text { in } X_{0} .\right.
$$

From the relation (3.29), we deduce that

$$
I_{\lambda}(w)=\lim _{n \rightarrow+\infty} I_{\lambda}\left(w_{n}\right)=\bar{c}<0 \quad \text { and } \quad I_{\lambda}^{\prime}(w)=0 .
$$

We conclude that $w$ is a nontrivial critical point of $I_{\lambda}$. Then $w$ is a nontrivial weak solution for problem $\left(\mathcal{P}_{K}\right)$.

Therefore, for any $\lambda \in\left(0, \lambda^{\star}\right)$ is an eigenvalue of problem $\left(\mathcal{P}_{K}\right)$.

The proof of Theorem 1.1 is complete.

Acknowledgements. I am very grateful to the reviewer for the careful reading of the paper and the valuable suggestions and constructive comments which helped to enrich the content and improve the presentation of the result in this paper.

\section{REFERENCES}

[1] A. Abdou and A. Marcos, Existence and multiplicity of solutions for a Dirichlet problem involving perturbed $p(x)-$ Laplacian operator, Electron. J. Differential Equations, 2016, (2016), no. 197, 19 pp.

[2] E. Azroul, A. Benkirane, M. Shimi, An introduction to generalized fractional Sobolev Space with variable exponent, Preprint. https://arxiv.org/abs/1901.05687. 
[3] E. Azroul, A. Benkirane, M. Shimi, Eigenvalue problems involving the fractional $p(x)-$ Laplacian operator, Adv. Oper. Theory, 4, (2019), no. 2, 539-555.

[4] A. Bahrouni and V. D. Rãdulescu, On a new fractional Sobolev space and applications to nonlocal variational problems with variable exponent, Discrete Contin. Dyn. Syst. Ser. S, 11, (2018), no. 3, 379-389.

[5] M. Bouslimi and K. Kefi, Existence of solution for an indefinite weight quasilinear prob-lem with variable exponent, Complex Var. Elliptic Equ., 58, ( 2013 ), no. 12, 1655-1666.

[6] L. Caffarelli, Diffusions, drifts and games, in nonlinear partial differential equations, Abel Symposia Springer, Berlin, 7, (2012) 37-52.

[7] L.Caffarelli, Surfaces minimizing non local energies, Atti Accad. Naz. Lincei Cl. Sci. Fis. Mat. Natur., 20, (2009), 281-299.

[8] L.Caffarelli,J.L.Vazquez, Non linear porous medium flow with fractional potential pressure, Arch.Ration.Mech.Anal., 202, (2011), 537-565.

[9] X.Chang, Z.Q.Wang, Ground state of scalar field equations involving a fractional Lapla- cian with general non linearity, Nonlinearity, 26, (2013), 479-494.

[10] M.Cheng, Bound state for the fractional Schrödinger equation with unbounded potential, J.Math.Phys., 53, (2012), 043507,7 pp.

[11] D.V. Cruz-Uribe, A. Fiorenza, Variable Lebesgue spaces. Foundations and harmonic analysis. Applied and Numerical Harmonic Analysis. Birkhäuser/Springer, Heidelberg, 2013.

[12] Edmunds, D., Rákosník, J., Sobolev embeddings with variable exponent, stud, Math., 143, (2000), $267-293$.

[13] X.L. Fan, Zhang. Q.H, Existence of solutions for $p(x)$-Laplacian Dirichlet problems, Nonlinear Anal. Theory Methods Appl., 52, (2003), 1843-1852.

[14] X. L. Fan, J. S. Shen, D.Zhao, Sobolev embedding theorems for spaces $W^{m, p(x)}(\Omega)$, J. Math. Anal. Appl., 262, (2001), 749 -760.

[15] Hichem Hajaie, Giovanni Molica Bisci , and Luca Vilasi, Existence results for a critical fractional equation, Asymptotic Analysis, 100, (2016), 209-225.

[16] I. Ekeland, On the variational principle, J. Math. Anal. Appl., 47, (1974), 324-353.

[17] U. Kaufmann, J. D. Rossi and R. Vidal, Fractional Sobolev spaces with variable exponents and fractional $p(x)$-Laplacians, Electronic J. Qual. Theory Differ. Equ., 2017, (2017), no.76, 10 pp.

[18] K. Kefi, $p(x)$-Laplacian with indefinite weight, Proc. Amer. Math. Soc., 139, (2011), no. 12, 4351-4360.

[19] O. Kovăcik \& J.Răkosník, On spaces $L^{p(x)}$ and $W^{k, p(x)}$, Czechoslovak Math. J., 41, (1991), 592-618.

[20] L.Silvestre, Regularity of the obstacle problem for a fractional power of the Laplace operator, Comm.Pure Appl.Math., 60, (2007) 67-112.

[21] N. Laskin, Quantum mechanics and Lévy path integrals, Phys. Lett. A, 268, (2000), 298-305.

[22] R. Metzler, J. Klafter, Restaurant at the random walk: recent developments in the description of anomalous transport by fractional dynamics, J. Phys. A, 37, (2004), 161-208.

[23] Nguyen Thanh Chung, Eigenvalue Problems for Fractional $p(x, y)$-Laplacian Equations with Indefinite Weight, Taiwanese Journal of mathematics, Vol. 23, No. 5, (October 2019), pp. 1153-1173.

[24] J.Tan, Y.Wang, J.Yang, Non linear fractional field equations, Nonlinear Anal., 75, (2012), 20982110.

[25] Wenjing Chen and Shengbing Deng, The Nehari manifold for nonlocal elliptic operators involving concave-convex, Z. Angew. Math. Phys., 2014, Springer Basel.

[26] R. Servadei, E. Valdinoci, Variational methods for nonlocal operators of elliptic type, Discrete and continuous dynamical systems, Volume 33, Number 5, , May 2013., pp. 2105- 2137.

[27] R. Servadei, E. Valdinoci, Lewy-Stampacchia type estimates for variational inequalities driven by nonlocal operators, Rev. Mat. Iberoam., 29, (2013), no. 3, 1091-1126.

[28] R. Servadei and E. Valdinoci, Pass solutions for nonlocal elliptic operators, J. Math. Anal. Appl., 389, (2012), 887-898.

[29] R. Servadei, E. Valdinoci, The Brezis-Nirenberg result for the fractional Laplacian., Trans. Amer. Math. Soc., 367, (2015), no. 1, 67-102.

[30] R. Servadei, E. Valdinoci, A Brezis-Nirenberg result for non-local critical equations in low dimension., Commun. Pure Appl. Anal., 12, (2013), no.6, 2445-2464.

Laboratory of Systems Engineering and Information Technologies (LISTI), National School of Applied

Sciences of Agadir, Ibn Zohr University, Morocco.

SAID TAARABTI: s.taarabti@uiz.ac.ma

Received 12/05/2020; Revised 27/08/2020 